\title{
Lung cancer mortality from exposure to chrysotile asbestos and smoking: a case-control study within a cohort in China
}

\author{
Eiji Yano, ${ }^{1}$ Xiaorong Wang, ${ }^{2}$ Mianzhen Wang, ${ }^{3}$ Hong Qiu, ${ }^{2}$ Zhiming Wang ${ }^{3}$
}

'Department of Hygiene and Public Health, Teikyo University School of Medicine, Tokyo, Japan

${ }^{2}$ School of Public Health and Primary Care, The Chinese University of Hong Kong, Hong Kong SAR, China

${ }^{3}$ Department of Occupational Health, Huaxi School of Public Health, Sichuan University, Chengdu, China

\section{Correspondence to}

Xiaorong Wang, School of Public Health and Primary Care, The Chinese University of Hong Kong, 4/F School of Public Health, Prince of Wales Hospital, Shatin, N.T., Hong Kong SAR, China; xrwang@cuhk.edu.hk

Accepted 5 April 2010 Published Online First 10 September 2010

\section{ABSTRACT \\ Objective To confirm the association between exposure to chrysotile asbestos and lung cancer risk and to demonstrate the combined effect of smoking and asbestos exposure. \\ Methods A case-control study of 1139 asbestos workers identified 41 male lung cancer cases in 2001; each case was matched by age ( \pm 5 years) with five controls. Workers in seven workshops were categorised into high-, medium- and low-exposure subgroups, and conditional logistic regression was applied to estimate the odds ratios for lung cancer risk associated with the different exposure levels. Smoking, age at first exposure, and exposure duration were considered as covariates/ confounding factors. A joint effect of asbestos exposure and smoking on lung cancer risk was analysed using a conditional logistical model.}

Results $54 \%$ of cases had high exposure and 24\% low exposure, while $24 \%$ of controls had high exposure and $44 \%$ low exposure. Smoking was more common in cases $(90 \%)$ than in controls (73\%). The adjusted OR for lung cancer was 3.66 (95\% Cl 1.61 to 8.29) for high exposure and was elevated slightly for medium exposure (1.25; $95 \% \mathrm{Cl} 0.47$ to 3.31). Smoking was related to lung cancer risk (OR 3.33; 95\% Cl 1.10 to 10.08). In comparison with the low-exposure non-smoking group, the $\mathrm{OR}$ for the high-exposure smoking group was 10.39 (1.34 to 82.45), in contrast to 5.23 (0.50 to 54.58) for high-exposure non-smoking workers.

Conclusions These results confirm the strong association between exposure to chrysotile asbestos and lung cancer risk, and support an interactive effect of asbestos exposure and smoking which is more than additive.

\section{INTRODUCTION}

The carcinogenicity of asbestos is well documented. Many countries, including Japan, have banned the production and use of asbestos, whereas some other countries, including China, continue to produce and use huge amounts of chrysotile asbestos in construction materials. Even where banned, asbestos continues to create many problems. For example, although domestic asbestos production is negligible in Japan, the country has in the past imported more than 10 million tons of asbestos, most of which remains in buildings and other structures. Asbestos removal and site cleaning have placed an enormous burden on society. Moreover, because of the long latency of asbestos-related diseases, health problems may emerge long after actual exposure. For example, in Amagasaki, Japan

\section{What this paper adds}

- Differences in the carcinogenic potency of different asbestos fibre types are debated in the scientific community.

- There is growing agreement that exposure to chrysotile is associated with increased risk for lung cancer, but more evidence is needed concerning the strength of the association and its interaction with smoking.

- This nested case-control study conducted in textile workers suggests a strong association between exposure to chrysotile asbestos and lung cancer risk.

- The result also supports a synergistic effect of asbestos exposure and smoking that is more than additive.

- The study delivers a strong message to policy makers that exposure to chrysotile is dangerous.

in 2005, several cases of mesothelioma were found among residents living in the vicinity of a former asbestos plant. ${ }^{1}$ This prompted nationwide concern about asbestos, and many similar cases were subsequently discovered and reported from other areas of the country.

There has been growing agreement in the scientific community that exposure to chrysotile asbestos, which is the most common type of asbestos currently produced and used, is associated with an increased risk of lung cancer, ${ }^{2-5}$ although the relationship between exposure to chrysotile asbestos and mesothelioma remains contentious. ${ }^{67}$ To control exposure to remaining asbestos and to compensate those with health damage due to asbestos exposure, it is important to establish dose-response relationships between asbestos exposure and diseases. However, sufficient information to assess asbestos exposure is usually lacking.

We previously reported the results of a 25-year follow-up observational study of a fixed cohort of 515 Chinese male workers in an asbestos textile plant. We found excessive lung cancer mortality associated with exposure to chrysotile asbestos in these workers. ${ }^{8}$ In the current nested case-control study, we further investigated the relationship between lung cancer and different levels of exposure to chrysotile asbestos. The combined effect of smoking and asbestos was also examined. 


\section{STUDY SUBJECTS AND METHODS}

The study subjects and controls were selected from an open cohort of asbestos workers in an asbestos textile plant in China. Cohort recruitment began in January 1972 and was open to those working in the plant between that time and November 1996. The final cohort consisting of 1139 male workers was followed for the occurrence of lung cancer until 2001. Nearly half of the members entered the cohort in 1972 and thus were followed for 30 years. All of the members were actively working in the plant and had no signs of overt cardiopulmonary disease at the time of cohort entry. According to plant records, only chrysotile asbestos had been used to produce asbestos textiles, cement products, friction materials, rubber products and heatresistant materials in the plant since 1939. Although we were not able to obtain samples representative of all of the chrysotile historically used in the plant, available samples of chrysotile assessed in 2000 by x-ray diffraction analysis and analytical transmission electron microscopy revealed that amphibole contamination was very low $\left(<0.001 \%\right.$ tremolite fibre) ${ }^{8}$

By the end of 2001, a total of 196 workers in the cohort had died, with 64 of these deaths occurring during the last 5 years. Forty-one lung cancer cases were identified from death certificates. These workers with lung cancers were selected as cases, and each was matched by age at the time of death ( \pm 5 years) with five controls using the incidence density sampling method. ${ }^{9}$ This approach randomly selected controls from the original cohort, taking into account follow-up status, that is, controls were alive, and exposure status, that is, exposure for controls was only counted prior to the date of the case's death. Thus, 41 cases and 205 controls were included in the data analysis. The workers were divided into seven job types and further categorised into three exposure groups based on the dust/fibre concentrations measured at major workshops and locations where the employees usually worked: (1) high-exposure group, including workers in the raw material and textile (carding, spinning and weaving) sections; (2) medium-exposure group, including those in the rubber section, and with job titles of maintenance and cleaning; and (3) low-exposure group, including those in the cement section and administration. ${ }^{8}$ Assigning the job categories of maintenance and cleaning to medium exposure, and administration to low exposure was based on industrial hygiene parameters. Smoking information was collected by face-to-face interview during the follow-up period. Subjects were classified as non-smokers and ever smokers; the latter was defined as having smoked at least one cigarette per day for 6 months or longer. The study was approved by the Human Subject Committees of Teikyo University and Huaxi School of Public Health.

We applied conditional logistic regression to estimate the odds ratios for lung cancer risk associated with the different levels of exposure, as represented by the three exposure groups, where those working in cement and administration (low exposure) served as a reference group. The other covariates/confounding factors considered included smoking status, age when cases died or age at first exposure, and exposure duration. We also assessed the joint effect of asbestos exposure and smoking on lung cancer risk, using conditional logistical regression taking into account age and exposure duration. In this analysis, we created five dummy variables based on combinations of the three exposure levels with smoking status. We defined those who did not smoke and who had a low exposure level (ie, working in the cement shop or administration) as the reference group. Finally, we estimated an interaction between exposure to asbestos and cigarette smoking in these subjects by calculating a synergy index $(S)$ and its $95 \%$ CI. $S$ is defined as the ratio of the observed effects of combined exposure to the two agents to the sum of the estimated separate effect of each ${ }^{10}$ :

$$
\begin{aligned}
S & =\left(O R_{\text {joint effect of asbestos smoke }}-1\right) /\left[\left(O R_{\text {asbestos }}-1\right)\right. \\
& \left.+\left(O R_{\text {smoke }}-1\right)\right]
\end{aligned}
$$

In this analysis, $S$ is the ratio of the excess risk in the group of high-exposure smokers to the sum of the excess risks in the lowexposure smokers and high-exposure non-smokers. The odds ratio used in the formula was adjusted for age and exposure duration. The $95 \%$ CI of $S$ was calculated using the delta method described by Hosmer and Lemeshow. ${ }^{11}$ All statistical analyses were performed using SPSS 15.0 for Windows, and the synergy index and 95\% CI were calculated using an Excel sheet available at http://www.epinet.se. ${ }^{12}$

\section{RESULTS}

The average age in both cases and controls was 66 years. The age at first exposure and number of years working in the asbestos plant were comparable between the two groups (table 1). In the cases, $54 \%$ were classified as having high asbestos exposure, and $24 \%$ as having low exposure; in contrast, $24 \%$ and $44 \%$ of the controls were classified as having high and low exposure, respectively. The differences between the two groups were statistically significant. Smoking was significantly more

\begin{tabular}{|c|c|c|c|c|c|}
\hline \multirow[b]{2}{*}{ Characteristic } & \multicolumn{2}{|c|}{ Cases $(n=41)$} & \multicolumn{2}{|c|}{ Controls $(n=205)$} & \multirow[b]{2}{*}{ p Value* } \\
\hline & n (\%) & Mean (SD) & $\overline{n(\%)}$ & $\overline{\text { Mean (SD) }}$ & \\
\hline Age, years & & $66.4(7.8)$ & & $66.0(9.1)$ & 0.788 \\
\hline Age at first exposure, years & & $32.0(9.0)$ & & $30.3(8.3)$ & 0.178 \\
\hline Exposure group & & & & & 0.001 \\
\hline High exposure & $22(53.7)$ & & $49(23.9)$ & & \\
\hline Medium exposure & $9(22.0)$ & & $66(32.2)$ & & \\
\hline Low exposure & $10(24.4)$ & & $90(43.9)$ & & \\
\hline Exposure duration, years & & $24.8(7.7)$ & & $25.4(9.0)$ & 0.661 \\
\hline Smoking & $37(90.2)$ & & $149(72.7)$ & & 0.017 \\
\hline Asbestosis & & & & & 0.006 \\
\hline None & $25(61.0)$ & & $168(82.0)$ & & \\
\hline Asbestosis I & $14(34.1)$ & & $28(13.7)$ & & \\
\hline Asbestosis II & $2(4.9)$ & & $9(4.4)$ & & \\
\hline Death & $41(100.0)$ & & $43(21.2)$ & & $<0.001$ \\
\hline
\end{tabular}

Table 1 Basic characteristics of lung cancer cases and controls

${ }^{*}$ The $t$-test was used for continuous variables and the $\chi^{2}$ test for categorical variables. 
Table 2 Conditional logistic regression* of lung cancer risk

\begin{tabular}{ll}
\hline Parameter & OR (95\% CI) \\
\hline Exposure group & \\
$\quad$ Low exposure & 1.0 \\
$\quad$ Medium exposure & $1.25(0.47$ to 3.31$)$ \\
$\quad$ High exposure & $3.66(1.61$ to 8.29$)$ \\
Smoking & $3.20(1.06$ to 9.66$)$ \\
Exposure duration, years & $1.00(0.96$ to 1.05$)$ \\
\hline
\end{tabular}

*Analysis was performed in 41 lung cancer cases and 205 controls.

common in the cases (90\%) than in the controls (73\%). In addition, asbestosis was diagnosed in 16 cases (39\%) and 37 controls (19\%). All lung cancer cases had died, while 43 controls had died of non-malignant diseases.

Multivariate analysis revealed that the risk for lung cancer in the high-exposure group was over three times that in the reference group (OR 3.66; 95\% CI 1.61 to 8.29) (table 2), and the difference was statistically significant. Medium exposure was associated with a slightly increased risk, which was not statistically significant (OR 1.25; 95\% CI 0.47 to 3.31). Smoking was associated with a significantly higher risk for lung cancer, which was more than three times higher than that for nonsmoking. No effect of age or exposure duration was observed, and there was no association between age at first exposure and cancer risk. When the interaction term of asbestos exposure and smoking was placed into the model, the OR in relation to high exposure and smoking was 0.65 (95\% CI 0.05 to 8.06 ), in comparison with the combination low exposure and nonsmoking.

In the joint effect analysis, no case in the group of nonsmokers had medium exposure (table 3 ). In comparison with the reference group (low-level asbestos exposure and non-smoking), all of the groups showed increased risk for lung cancer. There was an exposure-response trend in smokers: those with high asbestos exposure had a 10-fold greater risk. Despite a wide range in the confidence intervals, the odds ratio reached statistical significance. Smokers with medium exposure had an OR of 4.3, similar to the risk in non-smokers with a high exposure level, and smokers with a low exposure level had an OR of 3.0, neither of which was significant. The results indicated a joint effect of exposure to asbestos and smoking, and the potential synergic effect is further illustrated in figure 1. The calculated synergy index $(S)$ was 1.55 (95\% CI 0.43 to 5.67), which implies that the combined effect of high exposure and smoking was greater than additive. However, statistical significance was not reached, possibly because of inadequate statistical power arising from the small numbers available for subgroup analysis.

\section{DISCUSSION}

The strength of the association between exposure to chrysotile asbestos and lung cancer remains a topic of interest in the

Table 3 Joint effect of exposure to asbestos and smoking on lung cancer risk*

\begin{tabular}{lll}
\hline Exposure group & $\begin{array}{l}\text { Smoking }(\mathbf{n}=\mathbf{1 8 9}), \\
\text { OR }(\mathbf{9 5 \%} \mathbf{C l})\end{array}$ & $\begin{array}{l}\text { Non-smoking }(\mathbf{n}=\mathbf{5 7}), \\
\text { OR }(\mathbf{9 5 \%} \mathbf{C I})\end{array}$ \\
\hline Low & $3.04(0.36$ to 25.71$)$ & 1.00 \\
Medium & $4.34(0.52$ to 36.53$)$ & - \\
High & $10.39(1.34$ to 82.45$)$ & 5.23 (0.50 to 54.58) \\
\hline
\end{tabular}

${ }^{*}$ Analysis was carried out with conditional logistic regression in 41 lung cancer cases and 205 controls, adjusting for exposure duration. Low exposure/non-smoking subjects served as the reference group. scientific community. In addition to workers occupationally exposed to asbestos, the general population are also at risk, as substantial amounts of commercial asbestos products (mainly chrysotile) remain in the environment in many countries. ${ }^{1} 13$ Globally, an estimated 125 million people are occupationally exposed to asbestos annually, and 90000 die each year from asbestos-related diseases. ${ }^{14}$ Even in countries with total bans on the use of asbestos, new cases of asbestos-related cancer are likely to continue to emerge because of the long latency between exposure and disease onset.

Our study used a nested case-control design to assess the association between lung cancer and exposure to chrysotile. Overall, the study subjects were heavily exposed to chrysotile asbestos. The total dust concentrations in the work areas always exceeded $3 \mathrm{mg} / \mathrm{m}^{3}$, and personal samples taken for fibre analysis exceeded 3 fibres $/ \mathrm{ml}$, except in the cement workshop, where the fibre count was very low. ${ }^{8}$ According to historical records, exposure levels were much higher during the 1940s and 1970s. Available samples of chrysotile revealed that amphibole contamination was very low. However, it should be pointed out that samples available for analysis may not have been fully representative of historical use of chrysotile at this plant. It was possible that contamination by amphiboles varied in previously used raw materials that were not analysed. This was indicated in our recent case report of mesothelioma from the same plant, where tremolite was found in lung tissue. ${ }^{15}$ Nevertheless, there was no doubt that chrysotile was the predominant type of asbestos to which workers were exposed in this plant. After categorising the workers into three exposure levels, we observed a 3.6-fold increased risk for lung cancer in those with high exposure, that is, those working in the raw material and textile sections, which was significantly higher than that observed in those with low exposure. This association was independent of smoking, although smoking was also found to be a strong risk factor. This result confirms the strong association of chrysotile asbestos with lung cancer.

The difference in carcinogenic potency among the various types of asbestos fibres has been debated in the scientific community for more than 20 years. Many arguments against the amphibole hypothesis have been presented, asserting that there is virtually no difference between the risks for lung cancer presented by the different fibre types. ${ }^{5} 716$ Evidence also exists that chrysotile, crocidolite and amosite have produced similar levels of lung tumours in animal inhalation experiments. ${ }^{2}$ Systematic reviews of the qualitative risks for asbestos-related

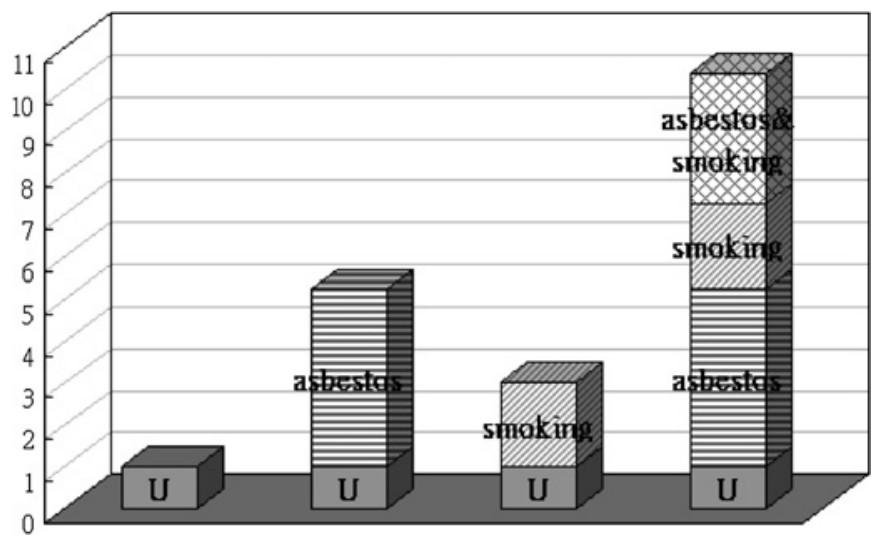

Figure 1 Odds ratios with contributions from different exposure categories. $U$ is the reference group of non-smokers with low-level asbestos exposure. 
diseases have suggested that fibre types may differ in their potency for causing mesothelioma, but the results for lung cancer were unclear, ${ }^{17}{ }^{18}$ suggesting that the amphibole hypothesis is not applicable to asbestos-related lung cancer. A recent study of North Carolina asbestos textile workers who were exposed to chrysotile also reported significantly higher than expected mortality from lung cancer, and an increased risk with cumulative exposure to asbestos fibres. ${ }^{5}$ On the other hand, studies in chrysotile cohorts have produced a wide range of risk estimations, for example, a low risk was estimated for Quebec chrysotile mine workers ${ }^{19}$ and a high risk estimation was reported for South Carolina textile workers. ${ }^{4}$ Similar heterogeneity in risk estimations and exposure-response associations was noted in asbestos textile workers who used the same form of asbestos. ${ }^{7} 1720$ Exposure level may be one of the factors determining the magnitude of risk. Asbestos-related diseases, including lung cancer, have often been clearly evident in workers with heavy chrysotile exposures. ${ }^{17}$ This may be attributable to the greater solubility of chrysotile in the lung, which may also partly explain the positive association observed in the present study. It should be noted that our control group was also exposed to asbestos. If the cases in the present study were to be compared with controls without asbestos exposure, and especially without smoking, the estimated risk for lung cancer might be even greater.

The joint effect of smoking and asbestos exposure on lung cancer risk has been documented. Considerable clinical and epidemiological evidence substantiates the fact that cigarette smoking exacerbates asbestos-induced lung cancer and that a large proportion of asbestos workers are also smokers. For example, more than $70 \%$ of the workers in the cohort from which the subjects were selected for the present study were smokers. Although a joint effect of smoking and asbestos is biologically plausible and observable, the form and magnitude of the combined effect remain inconclusive for certain conditions. Overall, evidence now indicates that the interaction of the two factors is more than additive. ${ }^{17}{ }^{21}$ It has been suggested that the combined effect of asbestos exposure and smoking reflects the facts that both factors are complex carcinogens, both can be genotoxic and cytotoxic, and both can affect more than one stage of lung carcinogenesis. ${ }^{22}$ The present study is consistent with a joint effect of high exposure and smoking greater than the sum of the individual effects, with a synergy index of 1.55 , supporting the finding that the excess lung cancer risk arising from the combination of asbestos exposure and smoking was greater than the sum of the two separate excess risks. ${ }^{23}$ Statistical significance, however, was absent. This might be due to inadequate statistical power in the subgroup analysis. Besides, using non-smokers without exposure to asbestos as a reference group might have resulted in a greater joint effect.

The major strength of this study was that unlike typical case-control studies, both the cases and controls were selected from the same cohort that had been followed from 1972 to 2001. Thus selection bias was not a major concern. The appearance of disease began to rapidly increase as the cohort aged. This study has a few limitations that need to be pointed out. One of them is the small number of cases, although each case was matched with five controls. Inadequate statistical power owing to small sample size was especially evident in the wide confidence intervals in the subgroup analysis determining the joint effect. Another limitation is that only about half of the lung cancer cases had pathological diagnoses, either by biopsy or autopsy. The remaining cases were diagnosed on the basis of CT and clinical course. Thus, there might have been some ambiguity in the diagnoses. Nevertheless, we believe that the proportion of misdiagnoses is small. The measure of smoking in this study was dichotomous, and detailed quantitative information on smoking was unavailable, which prohibited us from further determining an association with the extent of smoking. Data for asbestosis were available in this study. However, x-ray films taken before the 1980s were of poor quality. In addition, the ILO classifica$\operatorname{tion}^{24} 1 / 0$ is not classified as pneumoconiosis in the Chinese diagnostic criteria, which might have led to an underestimate of asbestosis in the subjects. Therefore, we did not include asbestosis in the multivariate analysis.

In summary, the present study confirms the strong association between exposure to chrysotile asbestos and risk for lung cancer, and supports a more-than-additive effect of exposure to asbestos and smoking. Given our reference group consisting of workers exposed to low levels of asbestos, the risk for lung cancer associated with exposure to chrysotile could be even greater if non-smokers without asbestos exposure were used as a reference group.

Funding This study was funded by Grants-in-Aid for Scientific Research (Scientific Research (B) 19390167) from the Japan Society for the Promotion of Science.

Competing interests None.

Patient consent Obtained.

Ethics approval This study was approved by the Human Subject Committees of Teikyo University and Huaxi School of Public Health, Sichuan University.

Provenance and peer review Not commissioned; externally peer reviewed.

\section{REFERENCES}

1. Kurumatani N, Kumagai S. Mapping the risk of mesothelioma due to neighbor-hood asbestos exposure. Am J Respir Crit Care Med 2008;178:624-9.

2. Stayner LT, Dankovic DA, Lemen RA. Occupational exposure to chrysotile asbestos and cancer risk: a review of the amphibole hypothesis. Am J Public Health 1996;86:179-86.

3. Cullen MR. Chrysotile asbestos: enough is enough. Lancet 1998;351:1377-8.

4. Hein MJ, Stayner LT, Leyman E, et al. Follow-up study of chrysotile textile workers: cohort mortality and exposure-response. Occup Environ Med 2007;64:616-25.

5. Loomis D, Dement JM, Wolf SH, et al. Lung cancer mortality and fibre exposures among North Carolina asbestos textile workers. Occup Environ Med 2009;66:535-42.

6. Mossman BT, Bignon J, Corn M, et al. Asbestos: scientific developments and implications for public policy. Science 1990;247:294-301.

7. Berman DW, Crump KS. A meta-analysis of asbestos-related cancer risk that addresses fiber size and mineral type. Crit Rev Toxicol 2008;38:49-73.

8. Yano E, Wang ZM, Wang XR, et al. Longitudinal study of the mortality of lung cancer in chrysolite workers. Am J Epidemiol 2001;154:538-43.

9. Richardson DB. An incidence density sampling program for nested case-control analyses. Occup Environ Med 2004;61:e59.

10. Rothman KJ. Epidemiology: an introduction. New York: Oxford University Press, 2002:168-80.

11. Hosmer DW, Lemeshow S. Confidence interval estimation of interaction. Epidemiology 1992;3:452-6.

12. Andersson T, Alfredsson L, Kallberg $\mathrm{H}$, et al. Calculating measures of biological interaction. Eur J Epidemiol. 2005;20:575-9 (Excel sheet for calculating biological interactions is available at http://www.epinet.se).

13. Magnani C, Agudo A, Gonzalez CA, et al. Multicentric study on malignant pleural mesothelioma and non-occupational exposure to asbestos. Br J Cancer 2000;83:104-11.

14. WHO. Elimination of asbestos-related diseases. World Health Organization, 2006 http://who.int/occupational_health/publications/asbestosrelateddiseases.pdf.

15. Yano E, Wang ZM, Wang XR, et al. Mesothelioma in a worker who spun chrysotile asbestos at home during childhood. Am J Ind Med 2009;52:282-7.

16. Nicholson WJ, Landrigan PJ. Asbestos: a status report. Curr Issues Public Health 1996:2:118-23.

17. Hodgson JT, Darnton A. The quantitative risks of mesothelioma and lung cancer in relation to asbestos exposure. Ann Occup Hyg 2000;44:565-601.

18. Berman DW, Crump KS. Update of potency factors for asbestos-related lung cancer and mesothelioma. Critical Rev Toxicol 2008;38:1-47.

19. Liddell FD, McDonald AD, McDonald JC. The 1891-1920 birth cohort of Quebec chrysotile miners and millers: development from 1904 and mortality to 1992. Ann Occup Hyg 1997;:41:13-36.

20. Stayner L, Smith R, Bailer J, et al. Exposure-response analysis of risk of respiratory disease associated with occupational exposure to chrysotile asbestos. Occup Environ Med 1997; 54:646-65. 
21. Lee PN. Relation between exposure to asbestos and smoking jointly and the risk of lung cancer. Occup Environ Med 2001;58:145-53.

22. Vainio H, Bofetta P. Mechanisms of the combined effect of asbestos and smoking in the aetiology of lung cancer. Scand J Work Environ Health 1994;20:235-42.
23. Rothman KJ. Synergy and antagonism in cause-effect relationships. Am J Epidemiol 1976;103:506-11.

24. ILO. Guidelines for the use of ILO international classification of radiographs of pneumoconioses. Rev edn. Geneva, Switzerland: International Labor Office. (Occupational Safety and Health Series 22). 1980. 